\title{
Catastrophic Injury and Its Association With Negative Mood States
}

\author{
Nirupama Wijesuriya, \\ Ashley Craig \\ The University of Sydney, \\ Sydney, Australia
}

\author{
Yvonne Tran \\ The University of \\ Sydney/University of Technology, \\ Sydney, Sydney, Australia
}

\author{
James Middleton \\ The University of Sydney, \\ Sydney, Australia
}

\begin{abstract}
Negative mood states are reported to be prevalent symptoms following SCI (spinal cord injury), however, controlled research primarily investigating its occurrence in SCI is uncommon. The primary objective of this paper was to study levels of negative mood states, such as fatigue and related states in people with SCI. Fatigue and mood states were assessed in people with SCI using validated measures, with comparisons made to able-bodied controls with similar age and sex ratio. Participants included 27 people with SCI living in the community and 27 able-bodied controls. The SCI group was found to have significantly elevated fatigue, as well as elevated levels of anger and reduced vigor. The risk of a person with SCI having elevated levels of fatigue was over four times than that of the able-bodied controls and their risk of having elevated anger was nine times more than that of able-bodied persons. The data presented in this study provide preliminary evidence that SCI is associated with negative mood states such as elevated fatigue and the clinical consequences of elevated fatigue to effective adjustment to SCI need to be explored.
\end{abstract}

Keywords: fatigue, anger, SCI (spinal cord injury), neurotrauma, negative mood

\section{Introduction}

Fatigue is associated with many chronic disorders as well as being associated with normal everyday functioning (Shen, Barbera, \& Shapiro, 2006). Unfortunately, there is a lack of a "gold standard" definition of what constitutes fatigue (Craig, Tran, Wijesuriya, \& Boord, 2006; Shen et al., 2006), resulting in terms, such as fatigue, sleepiness and drowsiness, often being used interchangeably (Wijesuriya, Tran, \& Craig, 2007). However, fatigue is defined that it is a complex phenomenon involving physical, psychosocial and behavioral processes (Shen et al., 2006; Wijesuriya et al., 2007). Fatigue includes feelings of tiredness or drowsiness and is known to involve performance decrements resulting from reduced alertness that impairs both capability and willingness to perform a task (Grandjean, 1988), as well as reduced cognitive and psychomotor skills (Craig et al., 2006). However, fatigue is not simply feeling tired or drowsy. It is known that drowsiness/tiredness is

\footnotetext{
*Acknowledgments: This research was supported by a grant from the New South Wales Premier SCI Grant (RIPG4) and by a grant from the Australian Research Council (LP0560590).

Nirupama Wijesuriya, Rehabilitation Studies Unit, Northern Clinical School, Faculty of Medicine, The University of Sydney.

Ashley Craig, Ph.D., Rehabilitation Studies Unit, Northern Clinical School, Faculty of Medicine, The University of Sydney.

Yvonne Tran, Ph.D., Rehabilitation Studies Unit, Northern Clinical School, Faculty of Medicine, The University of Sydney; Key University Centre for Health Technologies, University of Technology, Sydney.

James Middleton, Ph.D., Rehabilitation Studies Unit, Northern Clinical School, Faculty of Medicine, The University of Sydney.
} 
related to factors such as time of the day or an individual's sleep debt. Drowsiness occurs between an awake state and sleep state, and is believed to occur just before the onset of stage one micro-sleep (Broughton \& Hasan, 1995). Fatigue is also different to sleepiness, which has been defined specifically as an individual's inclination to sleep, or the difficulty remaining awake even while carrying out activities (Dement \& Carskadon, 1982; Shen et al., 2006) and is primarily related to circadian rhythm and homeostatic influences (Philip, Sagaspe, Moorec, Taillard, Charles, \& Guilleminault, 2005).

While fatigue is thought to be associated with subjective aspects of drowsiness or tiredness, research findings have shown that anxiety, depressive mood and negative sympathetic arousal are significantly associated with fatigue (Craig et al., 2006; Wijesuriya et al., 2007). Furthermore, research has suggested that fatigue can be explained by a two-level processing model in which physiological factors tap into lower level autonomic processing, while negative psychological factors such as anxiety tap into higher-level "executive" cognitive processes (Craig et al., 2006; Verwey \& Zaidel, 2000; Wijesuriya et al., 2007). Based on the above evidence, we defined fatigue for the purposes of this study as: A chronic state of tiredness or drowsiness that involves a complex interaction between autonomic, physiological and psychological factors, experienced and perceived as a negative state of mind associated with decrements in behavioral performance.

Research has shown that fatigue is high in people with neurotrauma. For instance, fatigue is elevated in people with traumatic brain injury, with one study suggesting almost $70 \%$ of people with brain injury report problems with significant fatigue (Ouellet \& Morin, 2006). SCI (spinal cord injury) is a neurological disorder in which the cord is severely injured by trauma or damaged as a result of disease. It is debilitating with severe impacts on lifestyle (Craig, Tran, \& Middleton, 2009; Kennedy \& Rogers, 2000) and has been found to reduce QOL (quality of life) substantially in domains, such as physical functioning and role, general health, vitality, as well as social, emotional and mental health functioning (Middleton, Tran, \& Craig, 2007; Westgren \& Levi, 1998). Research has also shown that QOL is reduced even further if a person with a neurological disorder has a history of chronic pain, or if the person has poor self-efficacy (Middleton et al., 2007; Schwartz, Coulthard-Morris, \& Zeng, 1996). The consequence of SCI is usually severe in terms of associated impact on mental health, with up to $40 \%$ at risk of suffering psychopathology, such as major depressive disorder, post-traumatic stress disorder and generalised anxiety disorder (Craig et al., 2009). Those living with SCI have also been shown to have over seven times the risk of having increased levels of depressive mood compared to able-bodied controls (Craig, Tran, \& Middleton, 2008). It has been suggested that risks of negative psychological states remain high unless people with SCI receive effective treatment such as cognitive behavior therapy during rehabilitation (Craig, 2008). Additional common co-morbid conditions include sleep disturbance, poor energy and appetite change (Krause, Bombardier, \& Carter, 2008).

Fatigue is believed to be a significant problem in SCI, even though research into SCI-related fatigue is limited. Richardson and Richards (2008) found sleep disturbance, fatigue and appetite disturbance to be consistent negative factors associated with reduced life satisfaction in the long-term following a SCI. Thompson (1999) studied activities of daily living and needs for assistance in adults with SCI attending an outpatient SCI clinic. They found that fatigue was the most frequent problem followed by pain and physical weakness. Fawkes-Kirby, Wheeler, Anton, Miller, Townson, and Weeks (2008) found that almost 60\% of outpatients with SCI had elevated levels of fatigue severe enough to interfere with daily function. Hammell, Miller, Forwell, Forman, and Jacobsen (2009) found fatigue, pain, depression, side effects of medications, poor quality sleep and spasticity to be the most serious conditions for people with SCI. Given the limited exploration 
into fatigue following SCI, the objective of the research presented in this paper was to conduct controlled research into fatigue in people with SCI. A second aim was to investigate the levels of other negative mood states such as anger.

\section{Method}

\section{Participants}

SCI participants were recruited from a Sydney SCI rehabilitation unit or through advertising for participants in a SCI community group newsletter. Able-bodied control participants were recruited through community advertising. Altogether, 27 adult persons were recruited (26 males, one female) who had chronic SCI, with a mean age of 50 years $(S D=9)$. The mean time since injury was 20.8 years $(S D=14)$. Exclusion criteria included a history of psychopathology before the SCI, prior traumatic brain injury and non-English speaking. Twenty participants had paraplegia or injury to the thoracic and lumbar regions (11 between T1-T12 and nine between L1-L5), while the remaining seven had tetraplegia or injury to the cervical region of the spine (three between $\mathrm{C} 3$ to $\mathrm{C} 5$ levels and four at either $\mathrm{C} 6$ or $\mathrm{C} 7$ levels). The able-bodied controls consisted of 27 adult persons who were similar to the SCI group for sex ratio (three females) and age (mean age $=46$ years, $S D=13$ ). The same exclusion criteria applied for the able-bodied controls. Participants were admitted into the study after they gave written consent. Institutional ethics approval was obtained prior to their participation in the study.

\section{Measures of Fatigue and Mood States}

The CFS (Chalder Fatigue Scale) (Chalder et al., 1993) was used to assess self-reported fatigue. This scale has been used frequently in fatigue research (Craig et al., 2006) and provides a reliable estimate of a person's mental and physical fatigue as well as an overall measure of fatigue. The scale has been shown to have high reliability and validity (Chalder et al., 1993). The POMS (profile of mood states) (McNair, Lorr, \& Droppleman, 2005) was also used to estimate the level of fatigue in the two groups as well as five other negative mood states, including tension, depressive mood, anger, vigor and confusion, and a total POMS score is also provided. The POMS has been shown to be a reliable and valid instrument in measuring psychological states and risk of psychopathology (McNair et al., 2005). Inspection of the items of the POMS suggests that it will provide estimates of negative mood states free of medical conditions that will be prevalent in a disorder like SCI likely to inflate the negative mood score (such as problems of sleeping, or reduced physical activity). Even though the POMS is used widely in non-SCI fields, it has rarely been used to estimate fatigue in people with SCI. Therefore, we believe the use of the POMS will make a valuable contribution to SCI research.

\section{Procedure}

All $27 \mathrm{SCI}$ and 27 able-bodied participants were approached to take part in the present study, while they were participating in an ongoing larger study into the relationship between brain activity and SCI. All participants were assessed in a temperature controlled stress-free laboratory. As part of the larger study, they were asked to complete a regimen of psychological tests, including the CFS and POMS scales. All participants completed the psychological tests before they completed the brain activity assessment.

\section{Analysis}

MANOVA (multivariate analysis of variance) was performed to determine overall differences between groups. If the MANOVA was significant, ANOVA (analyses of variance) were performed to determine where differences occurred. Eta-squared $\left(\eta^{2}\right)$ values are provided as estimates of the size of the difference between the 
groups. A $\eta^{2}$ around 0.03 is small, 0.13 represents a medium sized difference and over 0.2 represents a large difference (Cohen, 1988). The association between fatigue and the demographic factors was calculated using the Pearson correlation coefficient statistic and the relationship between fatigue and SCI was further described by $\chi^{2}$ analyses. The OR (odds ratio) was used to provide an estimate of the risk of having elevated fatigue as a function of SCI. The OR calculates the likelihood (odds of an event occurring in the SCI group to the likelihood of it occurring in the control group). An OR of one suggests that it is equally likely that both groups will have elevated fatigue, while an OR greater than one suggests elevated risks. The OR was calculated by the following formula: [(true positives)(true negatives)]/[(false positives)(false negatives)]. All analyses were performed using statistical software (Version 9, Statsoft).

\section{Results}

Overall significant differences were found between the two groups for the POMS factors: Wilks Lambda $=$ $0.68, F_{(7,46)}=3.1, p<0.01(0.01), \eta^{2}=0.32$, power $=90 \%$. Table 1 shows the descriptive statistics for the POMS mood states and CFS fatigue measures, as well as effect sizes $\left(\eta^{2}\right)$, ANOVA $F$-values and significance levels for the difference between groups for all measures. Inspection of Table 1 shows that the participants with SCI had significantly elevated scores for the POMS fatigue measure as well as for anger and the total POMS score. The SCI group also had significantly decreased vigor scores. Effect sizes for these three factors were moderate. POMS depressive mood and confusion scores were close to significance. Overall significant differences were also found for the CFS scores: Wilks Lambda $=0.88, F_{(2,51)}=3.4, p<0.05, \eta^{2}=0.12$, power $=$ $61 \%$. The SCI sample had significantly elevated CFS physical and total fatigue scores with moderate effect sizes, while a non-significant trend existed for the SCI to have raised levels of mental fatigue.

Table 1

Shows Statistics for the Fatigue and Negative Mood States for the SCI and Able-Bodied (AB) Groups. Effect Sizes, F-values and Significance Levels Are Shown

\begin{tabular}{|c|c|c|c|c|c|}
\hline Variable & $\begin{array}{l}\text { SCI } \\
\text { Mean }(S D) \pm 95 \mathrm{CI}\end{array}$ & $\begin{array}{l}A B \\
\text { Mean (SD) } \pm 95 \mathrm{CI}\end{array}$ & $\eta^{2}$ & $F_{(1,52)}$ & $p$ \\
\hline Tension & $5.9(5.6) 3.7-8.1$ & $4.9(3.9) 3.5-6.3$ & 0.01 & 0.61 & 0.44 \\
\hline Depressive & $7.6(10.3) 3.6-11.7$ & 3.6 (4.1) 2.0-5.2 & 0.06 & 3.5 & 0.06 \\
\hline Anger & $7.8(8.7) 4.4-11.3$ & 3.9 (4.0) $2.3-5.5$ & 0.07 & 4.4 & 0.04 \\
\hline Vigor & $18.7(5.1) 16.7-20.8$ & 22.5 (4.3) 20.8-24.3 & 0.14 & 8.8 & 0.01 \\
\hline Fatigue & $6.9(5.7) 4.7-9.2$ & 4.1 (2.9) 2.9-5.3 & 0.09 & 5.2 & 0.03 \\
\hline Confusion & 5.5 (4.1) 3.9-7.1 & 3.7 (2.7) 2.7-4.8 & 0.06 & 3.4 & 0.07 \\
\hline POMS total & 15.0 (31) 2.7-27.4 & $-2.3(16)-8.5-3.9$ & 0.11 & 6.6 & 0.01 \\
\hline CFS physical & 7.7 (4.5) 5.9-9.5 & 4.9 (3.2) 3.7-6.2 & 0.11 & 6.6 & 0.02 \\
\hline CFS mental & 3.9 (2.4) 2.9-4.9 & $3.1(2.3) 2.2-4.0$ & 0.03 & 1.6 & 0.20 \\
\hline CFS total & $11.6(6.4) 9.1-14.2$ & $8.1(4.9) 6.1-10.0$ & 0.09 & 5.1 & 0.03 \\
\hline
\end{tabular}

Notes. $S D=$ Standard deviation; $C I=$ Confidence interval; $A B=$ Able-bodied; $p=$ Probability.

Significant Pearson correlations were found between all CFS fatigue measures and the POMS mood state domains, with correlations ranging from 0.4 to $0.8(p<0.05)$. There were no significant associations found between CFS fatigue measures and demographic measures, such as age, level of injury and time since injury. Table 2 shows $\chi^{2}$ data in which participants who reported abnormally high levels of fatigue (CFS total fatigue) were compared to those with lower fatigue levels in both groups. A score of ten in the CFS total fatigue score 
was used as the cut-off score given that scores of ten constitute elevated fatigue (Craig et al., 2006). Persons with SCI had a significantly higher risk of having elevated fatigue $\left(\chi^{2}=6.03, d f=1, p<0.05\right)$, with an odds ratio score of 4.04. That is, the risk of persons with SCI having elevated fatigue is over four times than that of able-bodied persons. Anger can be an understandable reaction to sustaining a SCI (Middleton \& Craig, 2008). The SCI had significantly elevated anger scores compared to the able-bodied controls, and Table 3 shows $\chi^{2}$ data in which participants who reported abnormally high levels of anger (POMS anger) were compared to those with lower anger scores in both groups. A score of 14 in the POMS anger score was used as the cut-off score (given this score represents a significantly elevated anger score). Persons with SCI had a significantly higher risk of having elevated anger $\left(\chi^{2}=8.0, d f=1, p<0.01\right)$, with an odds ratio score of 9.1, that is, the odds of a person with SCI having a high level of anger is over nine times than that of an able-bodied person.

Table 2

SCI Participants Who Reported High Levels of Fatigue Versus Those With Low Levels Relative to the Able-Bodied Controls

\begin{tabular}{llll}
\hline Group & CFS low fatigue $(<10)$ & High CFS fatigue $(\geq 10)$ & Total \\
\hline SCI & 10 & 17 & 27 \\
AB controls & 19 & 8 & 27 \\
\hline
\end{tabular}

Notes. $\chi^{2}=6.03 ; d f=1 ; p<0.05 ;$ Odds ratio $=4.04 ; 95 \% \mathrm{CI}=1.3-12.6$.

Table 3

SCI Participants Who Reported High Levels of Anger Versus Those With Low Levels Relative to the Able-Bodied Controls

\begin{tabular}{llll}
\hline Group & POMS anger $(<14)$ & POMS anger $(\geq 14)$ & Total \\
\hline SCI & 20 & 7 & 27 \\
AB controls & 26 & 1 & 27 \\
\hline
\end{tabular}

Notes. $\chi^{2}=8.0 ; d f=1 ; p<0.01 ;$ Odds ratio $=9.1 ; 95 \% \mathrm{CI}=1.0-80.1$.

\section{Discussion and Conclusions}

This paper presents preliminary data that suggests people with SCI have increased risks of elevated fatigue, anger and reduced vigor, compared with an able-bodied group with a similar age and sex ratio. The SCI group was much more likely to suffer from physical fatigue, though there was a trend for them also to have elevated mental fatigue. The SCI group had over four times the risk of having elevated fatigue compared to the able-bodied controls ( $63 \%$ of the SCI sample had elevated levels of fatigue compared to $29 \%$ of the controls). Worryingly, people with SCI had over nine times the risk of having increased levels of anger or irritability, compared to the able-bodied controls ( $26 \%$ of the SCI people had elevated levels of anger compared to $4 \%$ of the controls). A trend also occurred for the SCI group to have elevated levels of confusion and depressive mood (Craig et al., 2009).

The SCI group was shown to have elevated fatigue (as defined in this paper) using two different self-report approaches to assessing severity. The POMS assesses fatigue by requesting participants to rate how they feel in a scale from 0 ("Not at all") to 4 ("Very much so"), for a number of descriptors, such as "tired", "lively", "sleepy", "sad" and so on. Arguably, this method should reduce possible mood state inflation due to items related to the effects of hospitalization or the injury rather than fatigue. Alternatively, the CFS employs a 
14-item approach, for example, "Do you have problems with tiredness?", "Do you have difficulty concentrating?" and "Have you lost interest in the things you used to do?". Given that the SCI participants were found to have elevated fatigue using both methods of assessment strengthens the likelihood that elevated fatigue associated with $\mathrm{SCI}$ is a valid finding.

As stated above, fatigue can be defined as a negative psychological state characterized by chronic tiredness and lethargy, highly associated with elevated anxiety and depressive mood (Craig et al., 2006). In other words, being fatigued is not a pleasant sensation. Consequently, this susceptibility to have a chronic fatigued state, in addition to feeling angry, represents a considerable obstruction to achieving positive life outcomes. A major focus of SCI rehabilitation is to enhance the person's capacity to adjust to the losses and subsequent difficulties of living with a SCI (Middleton \& Craig, 2008). The presence of elevated fatigue (and anger) will act as a barrier, effectively reducing the person's physical and mental capacity to adjust positively to the many challenges that arise as a result of the injury (Middleton \& Craig, 2008).

Given that the mean time, these results are concerning, since the injury was considerable, that is, just over 20 years. Adjustment has been found to decrease with increased time since the injury (Krause \& Crewe, 1991), though Dijkers (1999) found life satisfaction improved with time since injury. It should be expected that differential changes in health will occur over time, for instance, decreased vitality with increasing age. Notwithstanding the above, the findings of this research suggest that people with SCI continue to experience challenges related to their injury and impairment. The dynamics involved will need to be clarified by future research so that rehabilitation strategies can be developed that better target community living and long-term challenges of living with SCI (Middleton \& Craig, 2008). In summary, fatigue, as a problem, has not received significant research or clinical attention by rehabilitation health professionals in either the newly injured or with those attending outpatient clinics. Consequently, fatigue is not commonly assessed or considered as a primary risk of poor adjustment following SCI. Prospective research needs to be conducted to clarify the gaps in our knowledge about the interaction of fatigue with SCI, as well as the potential negative impact fatigue that may have on rehabilitation outcomes. The findings of such research could well lead to improved rehabilitation services that address the psychosocial needs of people with SCI (Craig \& Nicholson Perry, 2008).

\section{References}

Broughton, R., \& Hasan, J. (1995). Quantitative topographic electroencephalographic mapping during drowsiness and sleep onset. Journal of Clinical Neurophysiology, 12, 372-386.

Chalder, T., Berelowitz, G., Pawlikowska, T., Watts, L., Wessely, S., Wright, D., \& Wallace, E. P. (1993). Development of a fatigue scale. Journal of Psychosomatic Research, 37, 147-153.

Cohen, J. (1988). Statistical power analysis for the behavioral sciences. New Jersey: LEA.

Craig, A. (2008). Short and long-term outcomes following cognitive behavior therapy for people with SCI. In A. Craig, \& Y. Tran (Eds.), Psychological dynamics associated with spinal cord injury rehabilitation: New directions and best evidence. New York: Nova Science Publishers.

Craig, A., \& Nicholson Perry, K. (2008). Guide for health professionals on the psychosocial care for people with spinal cord injury. Sydney: New South Wales State Spinal Cord Injury Service.

Craig, A., Tran, Y., \& Middleton, J. (2008). The impact of spinal cord injury on psychological status. Proceedings of the 43rd APS Annual Conference (pp. 140-144). Hobart, September 23-28.

Craig, A., Tran. Y., \& Middleton, J. (2009). Psychological morbidity and spinal cord injury: A systematic review. Spinal Cord, 47, 108-114.

Craig, A., Tran, Y., Wijesuriya, N., \& Boord, P. (2006). A controlled investigation into the psychological determinants of fatigue. Biological Psychology, 72, 78-87. 
Dement, W. C., \& Carskadon, M. A. (1982). Current perspectives on daytime sleepiness: The issues. Sleep, 5, S56- S66.

Dijkers, M. P. J. M. (1999). Correlates of life satisfaction among persons with spinal cord injury. Archives of Physical Medicine and Rehabilitation, 80, 867-876.

Fawkes-Kirby, T. M., Wheeler, M. A., Anton, H. A., Miller, W. C., Townson, A. F., \& Weeks, C. A. O. (2008). Clinical correlates of fatigue in spinal cord injury. Spinal Cord, 46, 21-25

Grandjean, E. (1988). Fitting the task to the man. London: Taylor and Francis.

Hammell, K. W., Miller, W. C., Forwell, S. J., Forman, B. E., \& Jacobsen, B. A. (2009). Fatigue and spinal cord injury: A qualitative analysis. Spinal Cord, 47, 44-49

Kennedy, P., \& Rogers, B. (2000). Reported quality of life of people with spinal cord injuries: A longitudinal analysis of the first 6 months post-discharge. Spinal Cord, 38, 498-503.

Krause, J. S., \& Crewe, N. M. (1991). Chronologic age, time since injury, and time of measurement: Effect on adjustment after spinal cord injury. Archives of Physical Medicine and Rehabilitation, 72, 91-100.

Krause, J. S., Bombardier, C., \& Carter, R. E. (2008). Assessment of depressive symptoms during inpatient rehabilitation for spinal cord injury: Is there an underlying somatic factor when using the PHQ?. Rehabilitation Psychology, 53, 513-520.

McNair, D. M., Lorr, M., \& Droppleman, L. F. (2005). POMS: Profile of mood states manual. New York: MHS.

Middelton, J., \& Craig, A. (2008). Psychological challenges in treating persons with spinal cord injury. In A. Craig, \& Y. Tran (Eds.), Psychological dynamics associated with spinal cord injury rehabilitation: New directions and best evidence. New York: Nova Science Publishers.

Middleton, J., Tran, Y., \& Craig, A. (2007). Relationship between quality of life and self-efficacy in persons with spinal cord injuries. Archives of Physical Medicine and Rehabilitation, 88, 1643-1648.

O'Connor, P. J. (2006). Trends in spinal cord injury. Accident Analysis and Prevention, 38, 71-77.

Ouellet, M. C., \& Morin, C. M. (2006). Fatigue following traumatic brain injury: Frequency, characteristics, and associated factors. Rehabilitation Psychology, 51, 140-149.

Philip, P., Sagaspe, P., Moorec, N., Taillard, J., Charles, A., \& Guilleminault, C. (2005). Fatigue, sleep restriction and driving performance. Accident Analysis and Prevention, 37, 473-478.

Richardson, E. J., \& Richards, J. S. (2008). Factor structure of the PHQ-9 screen for depression across time since injury among persons with spinal cord injury. Rehabilitation Psychology, 53, 243-249.

Schwartz, C. E., Coulthard-Morris, L., \& Zeng, Q. (1996). Psychosocial correlates of fatigue in multiple sclerosis. Archives Physical Medicine Rehabilitation, 77, 165-170.

Shen, J., Barbera, J., \& Shapiro, C. M. (2006). Distinguishing sleepiness and fatigue: Focus on definition and measurement. Sleep Medicine Review, 10, 63-76.

Thompson, L. (1999). Functional changes in persons aging with spinal cord injury. Assistive Technology, 11, 123-129.

Verwey, W. B., \& Zaidel, D. M. (2000). Predicting drowsiness accidents from personal attributes, eye blinks and ongoing driving behavior. Personality and Individual Differences, 28, 123-142.

Westgren, N., \& Levi, R. (1998). Quality of life and traumatic spinal injury. Archives Physical Medicine and Rehabilitation, 79 , 1433-1439.

Wijesuriya, N., Tran, Y., \& Craig, A. (2007). The psychophysiological determinants of fatigue. International Journal of Psychophysiology, 63, 77-86. 